\title{
Research on the Stability of NDGM Model with the Fractional Order Accumulation and Its Optimization
}

\author{
Huiming Duan, ${ }^{1,2}$ Kailiang Shao, ${ }^{1}$ Xinping Xiao, ${ }^{2}$ and Jinwei Yang ${ }^{2}$ \\ ${ }^{1}$ College of Science, Chongqing University of Post and Telecommunications, Chongqing 400065, China \\ ${ }^{2}$ College of Science, Wuhan University of Technology, Wuhan 430070, China \\ Correspondence should be addressed to Xinping Xiao; xiaoxp@whut.edu.cn
}

Received 11 October 2016; Accepted 19 March 2017; Published 27 June 2017

Academic Editor: Francesco Braghin

Copyright (c) 2017 Huiming Duan et al. This is an open access article distributed under the Creative Commons Attribution License, which permits unrestricted use, distribution, and reproduction in any medium, provided the original work is properly cited.

\begin{abstract}
The grey forecasting model has been successfully applied in numerous fields since it was proposed. The nonhomogeneous discrete grey model (NDGM) was approximately constructed based on the nonhomogeneous index trend; it increased the applicability of discrete grey model. However, the NDGM required accurate data and better effect when the original data did not meet the conditions and fitting and prediction errors were larger. For this, the NDGM with the fractional order accumulating operator (abbreviated as $\mathrm{NDGM}^{p / q}$ ) has higher performance. In this paper, the matrix perturbation bound of the parameters was used to analyze the stability of $\mathrm{NDGM}^{p / q}$ and the $\mathrm{NDGM}^{p / q}$ can decrease the disturbance bound. Subsequently, the parameter estimation method of NDGM ${ }^{p / q}$ was studied and the Particle Swarm Optimization algorithm was employed to optimize the order number of $\mathrm{NDGM}^{p / q}$ and some steps were provided. In addition, the results of two practical examples demonstrated that the perturbation of $\mathrm{NDGM}^{p / q}$ was smaller than that of NDGM and provided remarkable predication performance compared with the traditional NDGM model and DGM model.
\end{abstract}

\section{Introduction}

Forecasting the future values of time series data plays a very important role in our research; thus, many forecasting methods have been developed for many years, such as the Rough sets theory proposed by Pawlak (see $[1,2])$ and fuzzy mathematics proposed by Zadeh (see [3]). However, because of limited knowledge and information, only part of system structure could be fully known. To address this problem, professor Deng proposed grey forecasting models to catch the system development tendency $[4,5]$.

As a core model of grey prediction, GM $(1,1)$ model has been widely used in some fields such as transportation, agriculture, economy, and management [6-10]. Meanwhile, many scholars have improved the GM $(1,1)$ model a lot [1119], thus enhancing its simulative accuracy and predictive accuracy. However, during the processes of both practical application and theoretical research of the GM $(1,1)$ model, GM $(1,1)$ model directly jumped from discrete form to continuous form, which resulted in failing in completely fitting homogeneous exponential sequence in simulation and prediction. Then the discrete grey model [20] was put forward to solve the transformation from discrete to continuous GM $(1,1)$ model. However, GM $(1,1)$ model and DGM model were constructed based on a hypothesis that the original data sequence was a homogeneous index sequence. However, the fact was not consistent, and the most original data sequence was the nonhomogeneous index sequence.

Hence, Xie and Liu [21] come up with nonhomogeneous discrete grey model (NDGM), and the model was constructed based on the approximate nonhomogeneous index trend. The results indicate there is no error between original value and simulative value based on pure nonhomogeneous index sequence. And NDGM model was the extension of DGM model while the latter was the special case of the former. NDGM model increased the applicability of discrete grey model. According to improve simulation and prediction accuracies, there were some the results $[22,23]$ of 
nonhomogeneous discrete grey model. However, these models had a higher requirement for data; when the data did not meet the requirements, the errors of both model-fitting and prediction were larger. The actual value cannot always meet the definition of monotonic increasing (decreasing) concave (convex) function. So the original data were accumulated to increase the exponent law according to the classical grey modeling mechanism. However, the accumulation of integer order was not suitable for some data, as modeling, and the effects of simulation and prediction were poor. Nevertheless, the current fraction grey model which used factional order accumulation [24-27] has the important significance for improving the performance of grey model.

Therefore, $\mathrm{Wu}$ et al. [28] defined the actual data with fractional order accumulation and defined the NDGM model with fractional order accumulation $\left(\mathrm{NDGM}^{p / q}\right)$. But they only presented the algorithm of model and some random fractional order values without considering the initial value of the model and presenting the nature of $\mathrm{NDGM}^{p / q}$ model. When a system was studied, the stability must be considered. They did not study the stability of the $\mathrm{NDGM}^{p / q}$ model; hence, the parameters of matrix perturbation bound were used to analyze the stability of $\mathrm{NDGM}^{p / q}$ model; then it was concluded that the solution of the $\mathrm{NDGM}^{p / q}$ model perturbation bound of solution was smaller than that of NDGM model. When $0<p / q<1$, the solution of $\mathrm{NDGM}^{p / q}$ model perturbation bound of solution was smaller and could decrease the disturbance bound. And they did not conclude which order number was the best as well as how to obtain the best order number and other properties. In order to solve these issues, the parameter estimation method of NDGM ${ }^{p / q}$ was studied. Furthermore, the Particle Swarm Optimization algorithm was employed to optimize the order number of the $\mathrm{NDGM}^{p / q}$ model and obtained the better simulation and prediction results. Finally, the results from previous works demonstrated that the perturbation bound solution of that $\mathrm{NDGM}^{p / q}$ model was smaller than traditional NDGM model in Case 1, and the results the Case 2 practical demonstrated that $\mathrm{NDGM}^{p / q}$ provided better predication performance than the traditional NDGM model and DGM model.

This paper is organized as follows. In Section 2, we introduced the NDGM model with integer order accumulating operator and the $\mathrm{NDGM}^{p / q}$ model. In Section 3, the stability of the NDGM ${ }^{p / q}$ model was discussed. In Section 4, the Particle Swarm Optimization algorithm was employed to optimize the order number of $\mathrm{NDGM}^{p / q}$ model; some steps were provided. Finally, in Section 5, the paper was concluded.

\section{NDGM Model with Fractional Order Accumulating Operator}

Definition 1 (see [29]). Assume that the sequence

$$
X^{(0)}=\left\{x^{(0)}(1), x^{(0)}(2), \ldots, x^{(0)}(n)\right\}
$$

is an original data sequence, and the symbol $D$ represents a kind of mathematical operational method. When $D$ is applied once on the sequence $X^{(0)}$, we have that

$$
X^{(0)} D^{(1)}=X^{(1)}=\left\{x^{(1)}(1), x^{(1)}(2), \ldots, x^{(1)}(n)\right\}
$$

is the accumulated generation sequence of $X^{(0)}$, where

$$
x^{(1)}(k)=\sum_{k=1}^{n} x^{(0)}(i), \quad k=1,2, \ldots, n .
$$

Then $D$ is called the first-order accumulating generation operator of $X^{(0)}$, denoted by 1-AGO. If $D$ is applied $r$ times on $X^{(0)}$, we obtain

$$
X^{(0)} D^{(r)}=X^{(r)}=\left\{x^{(r)}(1), x^{(r)}(2), \ldots, x^{(r)}(n)\right\},
$$

where

$$
x^{(r)}(k)=\sum_{k=1}^{n} x^{(r-1)}(i), \quad k=1,2, \ldots, n, r \in Z^{+} .
$$

Then $D$ is called the $r$-order accumulating generation operator of $X^{(0)}$, denoted by $r$-AGO.

Accordingly, the inverse accumulating generation operator is the inverse operation of the accumulating generation process and plays a role in recovery from the acts of accumulating operators; the inverse accumulating operator is defined as follows:

$$
\begin{aligned}
X^{(0)} D^{(r)} & =\alpha^{(r)} X^{(0)} \\
& =\left\{\alpha^{(r)} x^{(0)}(1), \alpha^{(r)} x^{(0)}(2), \ldots, \alpha^{(r)} x^{(0)}(n)\right\},
\end{aligned}
$$

where

$$
\begin{aligned}
\alpha^{(r)} x^{(0)}(k)=\left\{\alpha^{(r-1)} x^{(0)}(k)-\alpha^{(r-1)} x^{(0)}(\right. & k-1)\}, \\
k & =2,3, \ldots, n .
\end{aligned}
$$

In Definition 1, the order $r \in Z^{+}$, and we call the two operators accumulating and inverse accumulating generation operators with integer order.

The sequence

$$
Z^{(1)}=\left\{z^{(1)}(2), z^{(1)}(3), \ldots, z^{(1)}(n)\right\}
$$

is the mean sequence of $X^{(1)}$, where

$$
z^{(1)}(k)=\frac{1}{2}\left(x^{(1)}(k)+x^{(1)}(k-1)\right), \quad k=2,3, \ldots, n .
$$

Definition 2 (see [29]). The equation

$$
\begin{aligned}
x^{(0)}(k)+a z^{(1)}(k) & =b, \\
x^{(1)}(0) & =x^{(0)}(1)
\end{aligned}
$$

is called a $\operatorname{GM}(1,1)$ model, where $z^{(1)}(k)=(1 / 2)\left(x^{(1)}(k)+\right.$ $\left.x^{(1)}(k-1)\right), k=2,3, \ldots, n$. The whitenization equation $d x^{(1)} / d t+a x^{(1)}=b$ of $\operatorname{GM}(1,1)$ model is solved to obtain

$$
\widehat{x}^{(1)}(t+1)=\left(x^{(1)}-\frac{b}{a}\right) e^{-a t}+\frac{b}{a} .
$$


Definition 3 (see [20]). The sequences $X^{(0)}$ and $X^{(1)}$ are defined as (1) and (2). Then the equation

$$
x^{(1)}(k+1)=\beta_{1} x^{(1)}(k)+\beta_{2}
$$

is called discrete grey model (DGM). Similar with $\operatorname{GM}(1,1)$ model, $\beta_{1}$ and $\beta_{2}$ are the parameters of DGM model. The equation

$$
\begin{aligned}
\hat{x}^{(1)}(k+1)=\beta_{1}^{k}\left(x^{(0)}(1)-\frac{\beta_{2}}{1-\beta_{1}}\right) & +\frac{\beta_{2}}{1-\beta_{1}}, \\
k & =1,2, \ldots, n-1,
\end{aligned}
$$

is called the recursive function of DGM model.

Definition 4 (see [21]). The sequences $X^{(0)}$ and $X^{(1)}$ are defined as (1) and (2). Then the equation

$$
\begin{gathered}
\widehat{x}^{(1)}(k+1)=\beta_{1} \hat{x}^{(1)}(k)+\beta_{2} k+\beta_{3} \\
\hat{x}^{(1)}(1)=x^{(1)}(1)+\beta_{4}
\end{gathered}
$$

is called nonhomogenous discrete grey model (NDGM). $\hat{x}^{(1)}(k)$ is the simulative value of $x^{(1)}(k)$ and $x^{(1)}(1)$ is the iterative value of the NDGM model. $\beta_{1}, \beta_{2}, \beta_{3}$, and $\beta_{4}$ are parameters of NDGM model. The equation

$$
\widehat{x}^{(1)}(k+1)=\widehat{x}^{(1)}(1) \beta_{1}^{k}+\beta_{2} \sum_{j=1}^{k} j \beta_{1}^{k-j}-\frac{1-\beta_{1}^{k}}{1-\beta_{1}} \times \beta_{3}
$$

is called the recursive function of DGM model.

Definition 5. Let the $p / q(0<p / q<1)$ order accumulated generating operator of the original nonhomogeneous index sequence $X^{(0)}$ be $X^{(p / q)}$. Let $C_{p / q}^{0}=1, C_{k-1}^{k}=0$; then $x^{(p / q)}(k)=\sum_{i=1}^{k} C_{k-i+p / q-1}^{k-i} x^{(0)}(i), k=1,2, \ldots, n$, where

$$
\begin{aligned}
& C_{k-i+p / q-1}^{k-i} \\
& =\frac{(p / q+k-i-1)(p / q+k-i-2) \cdots(r+1)(p / q)}{(k-i) !} .
\end{aligned}
$$

$p / q$ order inverse accumulated generating operator of $X^{(0)}$ is

$$
\begin{gathered}
\alpha^{(p / q)} X^{(0)}=\alpha^{(1)} X^{(1-p / q)}=\left\{\alpha^{(1)} X^{(1-p / q)}(1),\right. \\
\left.\alpha^{(1)} X^{(1-p / q)}(2), \ldots, \alpha^{(1)} X^{(1-p / q)}(n)\right\} .
\end{gathered}
$$

Definition 6 (see [28]). Assume that

$$
\begin{aligned}
\widehat{x}^{(p / q)}(k+1) & =\beta_{1} \widehat{x}^{(p / q)}(k)+\beta_{2} k+\beta_{3} \\
\hat{x}^{(p / q)}(1) & =x^{(1)}(1)+\beta_{4}
\end{aligned}
$$

is established.

The sequences $X^{(0)}$ and $X^{(p / q)}$ are defined as (2) and (18). Then (18) is called nonhomogenous discrete grey model with fractional order accumulation (abbreviated as $\mathrm{NDGM}^{p / q}$ model). $\hat{x}^{(p / q)}(k)$ is the simulative value of $x^{(1)}(k)$ and $\widehat{x}^{(p / q)}(1)$ is the iterative value of the NDGM model. $\beta_{1}, \beta_{2}$, $\beta_{3}$, and $\beta_{4}$ are parameters of $\operatorname{NDGM}^{(p / q)}$ model.
That is least square method. So we can get the expressions of parameters in Proposition 7.

Proposition 7. Based on the least square method the first level parameters $\beta_{1}, \beta_{2}$, and $\beta_{3}$ satisfy the matrix equation

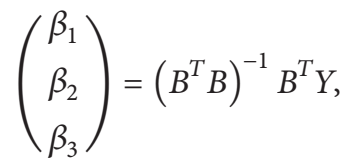

where

$$
B=\left(\begin{array}{ccc}
x^{(p / q)}(1) & 1 & 1 \\
x^{(p / q)}(2) & 2 & 1 \\
\vdots & \vdots & \vdots \\
x^{(p / q)}(n-1) & n-1 & 1
\end{array}\right) \text {, }
$$

$$
Y=\left(\begin{array}{c}
x^{(p / q)}(2) \\
x^{(p / q)}(3) \\
\vdots \\
x^{p / q}(n)
\end{array}\right) \text {. }
$$

Proposition 8. The recursive function of $N D G M^{p / q}$ model is

$$
\begin{array}{r}
\widehat{x}^{(p / q)}(k+1)=\beta_{1}^{k} \widehat{x}^{(p / q)}(1)+\beta_{2} \sum_{j=1}^{k} j \beta_{1}^{k-j}+\frac{1-\beta_{1}^{k}}{1-\beta_{1}} \beta_{3}, \\
k=1,2, \ldots, n-1 .
\end{array}
$$

Proof. By Definition 6, we have

$$
\begin{aligned}
& \widehat{x}^{(p / q)}(k+1)=\beta_{1} \widehat{x}^{(p / q)}(k)+\beta_{2} k+\beta_{3} \\
&= \beta_{1}\left(\beta_{1} \widehat{x}^{(p / q)}(k-1)+\beta_{2}(k-1)+\beta_{3}\right)+\beta_{2} k \\
&+\beta_{3} \\
&= \beta_{1}^{2} \widehat{x}^{(p / q)}(k-1)+\beta_{2}\left[\beta_{1}(k-1)+k\right] \\
&+\beta_{3}\left(1+\beta_{1}\right) \\
&= \beta_{1}^{3} \widehat{x}^{(p / q)}(k-2) \\
&+\beta_{2}\left[\beta_{1}^{2}(k-2)+\beta_{1}(k-1)+k\right] \\
&+\beta_{3}\left(1+\beta_{1}+\beta_{1}^{2}\right)=\ldots \quad k \quad k=1,2, \ldots, n-1 . \\
&= \beta_{1}^{k} \widehat{x}^{(p / q)}(1)+\beta_{2} \sum_{j=1}^{k} j \beta_{1}^{k-j}+\frac{1-\beta_{1}^{k}}{1-\beta_{1}} \beta_{3},
\end{aligned}
$$

We use the least square method to calculate the value of parameter $\beta_{4}$. By minimizing the error of $\hat{x}^{(p / q)}(k)$ and 
$x^{(p / q)}(k), \min _{\beta_{4}}=\sum_{k=1}^{n}\left[\widehat{x}^{(1)}(k)-x^{(1)}(k)\right]$. We can construct a nonrestraint optimized model; then

$$
\beta_{4}=\frac{\left[\widehat{x}^{p / q}(k+1)-\beta_{1}^{k} x^{(1)}(1)-\beta_{2} \sum_{j=1}^{k} j \beta_{1}^{k-j}-\beta_{3}\left(\left(1-\beta_{1}^{k}\right) /\left(1-\beta_{1}\right)\right)\right] \beta_{1}^{k}}{1+\sum_{k=1}^{n-1}\left(\beta_{1}^{k}\right)^{2}},
$$

where $k=1,2, \ldots, n-1$.

\section{The Stability of the Solution of NDGM Model with Fractional Order Accumulation}

3.1. The Stability of $N D G M^{p / q}$ Model. In order to illustrate the stability of the $\mathrm{NDGM}^{p / q}$ model, the theorem related to the matrix perturbation analysis was introduced as follows.

Lemma 9 (see [29]). Let $A \in C^{n \times n}, b \in C^{n}, M=A+E$, and $c=b+k$. Suppose $x+h$ and $x$ satisfy $\|M x-c\|_{2}=$ min and $\|A x-b\|_{2}=$ min. If $\operatorname{rank}(A)=\operatorname{rank}(M)$ and $\left\|A^{\dagger}\right\|_{2}\|E\|_{2}<1$, where $A^{\dagger}$ is the pseudo-inverse of matrix $A$, then

$$
\|h\| \leq \frac{\kappa^{\dagger}}{\gamma^{\dagger}}\left(\frac{\|E\|_{2}}{\|A\|}\|x\|+\frac{\|k\|}{\|A\|}+\frac{\kappa^{\dagger}}{\gamma^{\dagger}} \frac{\|E\|_{2}}{\|A\|} \frac{\left\|r_{x}\right\|}{\|A\|}\right)
$$

where $\|A\|,\|E\|$, and $\left\|r_{x}\right\|$ are the tolerance norm and $r_{x}=b-$ $A x, \kappa^{\dagger}=\left\|A^{\dagger}\right\|_{2}\|A\|$, and $\gamma^{\dagger}=1-\left\|A^{\dagger}\right\|_{2}\|E\|_{2}$.

Theorem 10. For the NDGM $M^{p / q}$ model of original data $\left\{x^{(0)}(1), x^{(0)}(2), \ldots, x^{(0)}(n)\right\}$ based on nonhomogeneous index sequence, if the rth data is disturbed, that is, $\hat{x}^{(0)}(r)=x^{(0)}(r)+$ $\varepsilon, r=1,2, \ldots, n-1$, based on the least square method $\|B X-Y\|=\min$, let $x$ be a solution of the Lemma 9; then the perturbation bound is $L_{1}\left(x^{(0)}(r)\right)$ and

$$
\begin{aligned}
L_{1}\left(x^{(0)}(1)\right)=|\varepsilon| \frac{\kappa^{\dagger}}{\gamma^{\dagger}} \frac{\sqrt{\sum_{k=1}^{n-1}\left(C_{k+p / q-2}^{k-1}\right)^{2}}}{\|B\|}\|x\| \\
+\frac{\sqrt{\sum_{k=2}^{n}\left(C_{k+p / q-2}^{k-1}\right)^{2}}}{\|B\|} \\
+\frac{\kappa^{\dagger}}{\gamma^{\dagger}} \frac{\sqrt{\sum_{k=1}^{n-1}\left(C_{k+p / q-2}^{k-1}\right)^{2}}}{\|B\|} \frac{\left\|r_{x}\right\|}{\|B\|}, \\
L_{1}\left(x^{(0)}(r)\right)=|\varepsilon| \frac{\kappa^{\dagger}}{\gamma^{\dagger}}, \\
+\frac{\sqrt{\sum_{k=1}^{n-r}\left(C_{k+p / q-2}^{k-1}\right)^{2}}}{\|B\|}\|x\|
\end{aligned}
$$

$$
+\frac{\kappa^{\dagger}}{\gamma^{\dagger}} \frac{\sqrt{\sum_{k=1}^{n-r}\left(C_{k+p / q-2}^{k-1}\right)^{2}}}{\|B\|} \frac{\left\|r_{x}\right\|}{\|B\|}
$$

where $r=2,3, \ldots, n-1$, and when $\hat{x}^{(0)}(n)=x^{(0)}(n)+\varepsilon$, then

$$
L_{1}\left(x^{(0)}(n)\right)=\frac{\kappa^{\dagger}}{\gamma^{\dagger}} \frac{|\varepsilon|}{\|B\|}
$$

where mathematical notation is the same as Lemma 9.

Proof. If $\varepsilon$ is regarded as a disturbance of $x^{(0)}(1)$, then

$$
\widehat{B}=B+\Delta B
$$$$
=\left(\begin{array}{ccc}
x^{(p / q)(1)} & 1 & 1 \\
x^{(p / q)}(2) & 2 & 1 \\
\vdots & \vdots & \vdots \\
x^{(p / q)}(n-1) & n-1 & 1
\end{array}\right)
$$$$
+\left(\begin{array}{ccc}
\varepsilon & 0 & 0 \\
\frac{p}{q} \varepsilon & 0 & 0 \\
\vdots & \vdots & \vdots \\
C_{n-3+p / q}^{n-2} & 0 & 0
\end{array}\right)
$$

$$
\widehat{Y}=Y+\Delta Y\left(\begin{array}{c}
x^{(p / q)}(2) \\
x^{(p / q)}(3) \\
\vdots \\
x^{(p / q)}(n)
\end{array}\right)+\left(\begin{array}{c}
\frac{p}{q} \varepsilon \\
C_{1+p / q^{\varepsilon}}^{2} \\
\vdots \\
C_{n-2+p / q}^{n-1} \varepsilon
\end{array}\right)
$$

thus, we have

$$
\begin{aligned}
& (\Delta B)^{T} \Delta B \\
& =\left(\begin{array}{cccc}
1+\left(\frac{p}{q}\right)^{2}+\left(C_{p / q+1}^{2}\right)^{2}+\cdots+\left(C_{n-2+p / q}^{n-1}\right)^{2} \varepsilon^{2} & 0 & 0 \\
0 & 0 & 0 \\
0 & 0 & 0
\end{array}\right) .
\end{aligned}
$$


Because $1+(p / q)^{2}+\left(C_{p / q+1}^{2}\right)^{2}+\cdots+\left(C_{n-2+p / q}^{n-1}\right)^{2} \varepsilon^{2}$ is $\Delta B^{T} \Delta B$ maximum of eigenvalue, then

$$
\begin{aligned}
\|\Delta B\|_{2} & =\sqrt{\lambda_{\max }\left(\Delta B^{T} \Delta B\right)}=\sqrt{\sum_{k=1}^{n-1}\left(C_{k+p / q-2}^{k-1}\right)^{2}}|\varepsilon| \\
\|\Delta Y\|_{2} & =\sqrt{\left(\frac{p}{q}\right)^{2}+\left(C_{p / q+1}^{2}\right)^{2}+\cdots+\left(C_{n-2+p / q}^{n-1}\right)^{2}}|\varepsilon| \\
& =\sqrt{\sum_{k=2}^{n}\left(C_{k+p / q-2}^{k-1}\right)^{2}}|\varepsilon| .
\end{aligned}
$$

By Lemma 9 and based on the least square method $\| B x-$ $Y \|_{2}=\min , \Delta x$ is the disturbed solution of equation $B x=Y$, and we have

$$
\begin{aligned}
& \Delta x \leq \frac{\kappa^{\dagger}}{\gamma^{\dagger}}\left(\frac{\|\Delta B\|_{2}}{\|B\|}\|x\|+\frac{\|\Delta Y\|}{\|B\|}+\frac{\kappa^{\dagger}}{\gamma^{\dagger}} \frac{\|\Delta B\|_{2}}{\|B\|} \frac{\left\|r_{x}\right\|}{\|B\|}\right), \\
& L_{1}\left(x^{(0)}(1)\right) \\
& =|\varepsilon| \frac{\kappa^{\dagger}}{\gamma^{\dagger}} \frac{\sqrt{\sum_{k=1}^{n-1}\left(C_{k+p / q-2}^{k-1}\right)^{2}}\|x\|}{\|B\|} \| \frac{\sqrt{\sum_{k=2}^{n}\left(C_{k+p / q-2}^{k-1}\right)^{2}}}{\|B\|} \frac{\left\|r_{x}\right\|}{\|B\|} . \\
& +\frac{\kappa^{\dagger}}{\gamma^{\dagger}} \frac{\sqrt{\sum_{k=1}^{n-1}\left(C_{k+p / q-2}^{k-1}\right)^{2}}}{\|B\|}
\end{aligned}
$$

When $\widehat{x}^{(0)}(2)=x^{(0)}(2)+\varepsilon$, then

$$
\begin{aligned}
& \Delta B=\left(\begin{array}{ccc}
0 & 0 & 0 \\
\varepsilon & 0 & 0 \\
\frac{p}{q} & 0 & 0 \\
\vdots & \vdots & \vdots \\
C_{n-4+p / q^{\varepsilon}}^{n-3} & 0 & 0
\end{array}\right), \\
& \Delta Y=\left(\begin{array}{c}
\varepsilon \\
\frac{p}{q} \varepsilon \\
C_{1+p / q}^{2} \\
\vdots \\
C_{n-3+p / q}^{n-2}
\end{array}\right)
\end{aligned}
$$

According to the proof of $L_{2}\left(x^{(0)}(1)\right)$, we have

$$
\begin{aligned}
L_{1}\left(x^{(0)}(2)\right)=|\varepsilon| \frac{\kappa^{\dagger}}{\gamma^{\dagger}} \frac{\sqrt{\sum_{k=1}^{n-2}\left(C_{k+p / q-2}^{k-1}\right)^{2}}}{\|B\|}\|x\| \\
+\frac{\sqrt{\sum_{k=1}^{n-1}\left(C_{k+p / q-2}^{k-1}\right)^{2}}}{\|B\|} \\
+\frac{\kappa^{\dagger}}{\gamma^{\dagger}} \frac{\sqrt{\sum_{k=1}^{n-2}\left(C_{k+p / q-2}^{k-1}\right)^{2}}}{\|B\|} \frac{\left\|r_{x}\right\|}{\|B\|} .
\end{aligned}
$$

According to this rule, if $\varepsilon$ is regarded as a disturbance of $x^{(0)}(r), r=3,4, \ldots, n-1$, both $\Delta B$ and $\Delta Y$ changed; thus,

$$
\begin{aligned}
L_{1}\left(x^{(0)}(r)\right)=|\varepsilon| \frac{\kappa^{\dagger}}{\gamma^{\dagger}} \frac{\sqrt{\sum_{k=1}^{n-r}\left(C_{k+p / q-2}^{k-1}\right)^{2}}}{\|B\|}\|x\| \\
+\frac{\sqrt{\sum_{k=1}^{n-r+1}\left(C_{k+p / q-2}^{k-1}\right)^{2}}}{\|B\|} \\
+\frac{\kappa^{\dagger}}{\gamma^{\dagger}} \frac{\sqrt{\sum_{k=1}^{n-r}\left(C_{k+p / q-2}^{k-1}\right)^{2}}}{\|B\|} \frac{\left\|r_{x}\right\|}{\|B\|} .
\end{aligned}
$$

If $\varepsilon$ is regarded as a disturbance of $x^{(0)}(n)$, we have

$$
\begin{aligned}
(\Delta B)^{T} \Delta B & =\left(\begin{array}{lll}
0 & 0 & 0 \\
0 & 0 & 0 \\
0 & 0 & 0
\end{array}\right), \\
Y & =\left(\begin{array}{c}
0 \\
0 \\
\vdots \\
\varepsilon
\end{array}\right), \\
L_{1}\left(x^{(0)}(n)\right) & =\frac{\kappa^{\dagger}}{\gamma^{\dagger}} \frac{|\varepsilon|}{\|B\|} .
\end{aligned}
$$

When $p / q=1$, let $L_{2}\left(x^{(0)}(r)\right.$ be the perturbation bound of the NDGM model. By Theorem 10, we have

$$
\begin{aligned}
& L_{2}\left(x^{(0)}(r)\right)=|\varepsilon| \frac{\kappa^{\dagger}}{\gamma^{\dagger}}\left(\frac{\sqrt{n-r}\|x\|}{\left\|B_{1}\right\|}+\frac{\sqrt{n-r+1}}{\left\|B_{1}\right\|}\right. \\
& \left.+\frac{\kappa^{\dagger}}{\gamma^{\dagger}} \sqrt{n-r} \frac{\left\|r_{x}\right\|}{\left\|B_{1}\right\|}\right), \quad r=1,2, \ldots, n-1, \\
& L_{2}\left(x^{(0)}(n)\right)=\frac{\kappa^{\dagger}}{\gamma^{\dagger}} \frac{|\varepsilon|}{\left\|B_{1}\right\|} .
\end{aligned}
$$


TABLE 1: The fitted values and MAPE of four grey models (unit: millionth).

\begin{tabular}{|c|c|c|c|c|c|}
\hline Year & Actual value & $\operatorname{GM}(1,1)$ & NDGM & $\mathrm{NDGM}^{0.5}$ & $\mathrm{NDGM}^{0.01}$ \\
\hline 2000 & 5.08 & 5.08 & 5.08 & 5.08 & 5.08 \\
\hline 2001 & 4.80 & 3.55 & 3.65 & 3.74 & 4.23 \\
\hline 2002 & 4.67 & 4.52 & 4.61 & 4.41 & 4.43 \\
\hline 2003 & 4.50 & 5.76 & 5.84 & 5.71 & 5.50 \\
\hline 2004 & 7.12 & 7.34 & 7.42 & 7.47 & 7.29 \\
\hline 2005 & 9.67 & 9.34 & 9.45 & 9.70 & 9.67 \\
\hline 2006 & 12.80 & 11.90 & 12.04 & 12.42 & 12.54 \\
\hline 2007 & 15.88 & 15.15 & 15.37 & 15.66 & 15.84 \\
\hline 2008 & 19.49 & 19.30 & 19.65 & 19.51 & 19.48 \\
\hline MAPE & & 10.01 & 8.93 & 8.01 & 5.49 \\
\hline 2009 & 23.07 & 24.57 & 25.13 & 24.02 & 23.42 \\
\hline 2010 & 26.86 & 31.29 & 32.16 & 29.28 & 27.59 \\
\hline MAPE & & 11.50 & 14.32 & 6.55 & 2.11 \\
\hline
\end{tabular}

Obviously, $\kappa^{\dagger} / \gamma^{\dagger}$ and $\|\Delta B\|$ are all positive and increasing function of $r$; other variables are not changed. Therefore, perturbation bounds $L_{1}\left(x^{(0)}(r)\right)$ and $L_{2}\left(x^{(0)}(r)\right)$ are the increasing function of the effect of sample size $n$; that is, $L_{1}\left(x^{(0)}(r)\right)$ and $L_{2}\left(x^{(0)}(r)\right)$ will change large when $n \rightarrow+\infty$. When $r$ is a constant value, $p / q$ is lager; then $L_{1}\left(x^{(0)}(r)\right)$ is lager too. When $0<p / q \leq 1$, we have $L_{1}\left(x^{(0)}(r)\right)<$ $L_{2}\left(x^{(0)}(r)\right), r=1,2, \ldots, n-1$. When the perturbation bound of solution was large, the perturbation was not always large. This was because the perturbation cannot exceed the perturbation bound. Hence, from the perspective of the size of perturbation bound, when $\widehat{x}^{(0)}(r)=x^{(0)}+\mathcal{E}, r=1,2, \ldots, n-$ 1 , the disturbed solution of the $\mathrm{NDGM}^{p / q}$ model is lower. The $\mathrm{NDGM}^{p / q}$ model is more stable than NDGM model.

3.2. Verification of the Stability NDGM ${ }^{p / q}$ Model. In this section, the effectiveness of the NDGM ${ }^{p / q}$ model is verified by two real cases study. Mean absolute percentage error (MAPE) compares the real and forecasted values to evaluate the precision. MAPE is defined as (MAPE $=100 \%(1 / n) \sum_{k=1}^{n} \mid(x(k)-$ $\widehat{x}(k)) / x(k) \mid)$, where $x^{(0)}(k)$ is the actual value at time $k$, and $\hat{x}^{(0)}(k)$ is the predicted value for time $k$.

Case 1 (syphilis incidence predicted in China [30]). The trends of syphilis incidence in China forecasting example [31] compare the precision; the historical incidence of China from 2000 to 2008 was employed as the model-fitting. Then the actual values of 2009 and 2010 were predicted. The $\mathrm{NDGM}^{p / q}$ model, NDGM model, and a classical GM $(1,1)$ model were built, respectively, to simulate the trends of syphilis incidence in China. The simulated/forecasted values and absolute percentage error with different grey models were shown in Table 1.

As can be seen from Table 1, from a short-term forecasting viewpoint, $\mathrm{NDGM}^{0.01}$ obtained lower MAPE than the $\operatorname{GM}(1,1)$ model, which implied that the $\mathrm{NDGM}^{0.01}$ could significantly enhance the precision of grey forecasting

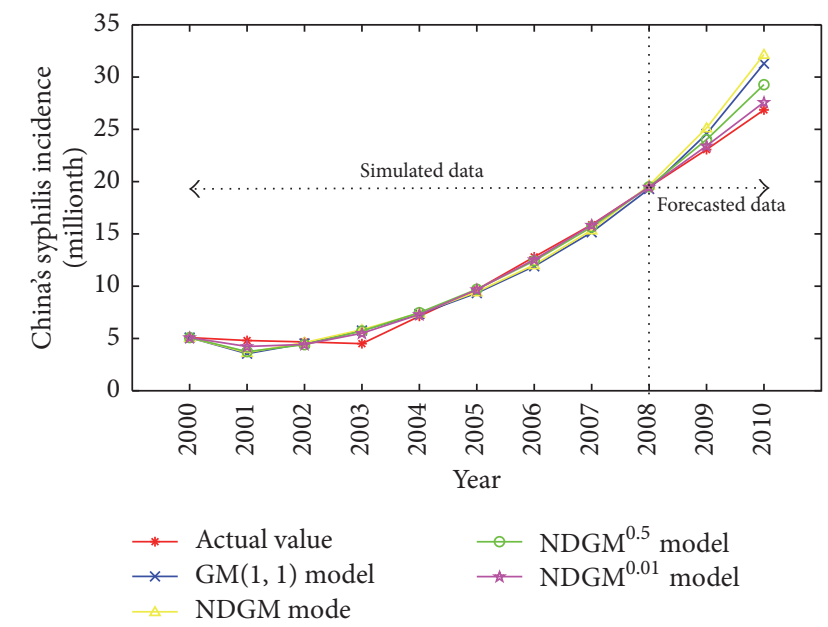

FIGURE 1: Simulates and forecasts obtained using different grey models.

model. Moreover, NDGM ${ }^{0.01}$ obtained lower MAPE than the $\mathrm{NDGM}^{0.5}$; therefore, it was also shown that the perturbation bound was smaller and the prediction accuracy was higher. According to Table 1, a scatter broken-line figure regarding the simulated and forecasted data was shown in Figure 1.

Figure 1 showed that the whole performances of the four models for simulating and forecasting the trends of syphilis incidence in China. Obviously, the prediction effect of GM(1, 1) model was better than that of NDGM model, but the simulation and prediction precisions of other NDGM models were better than those of GM(1,1) model. For NDGM model, the simulation and prediction precisions of $\mathrm{NDGM}^{0.01}$ were the best, followed by NDGM ${ }^{0.5}$, but those of NDGM model of integer order were the worst. Moreover, for the NDGM model of fractional order, the simulation, and prediction precisions of smaller fraction were higher than those of bigger one. In addition, Theorem 10 can also be proved to be correct from the stability of system. Namely, the smaller the perturbation 
order was, the higher the system stability was, and the higher the precisions of simulation and prediction were.

\section{Optimizing the Order Number of the NDGM ${ }^{p / q}$ Model by Particle Swarm Optimization (PSO) Algorithm}

4.1. $N D G M^{p / q}$ Model Steps. We have discussed the NDGM ${ }^{p / q}$ model and the main steps of modeling; the steps were as follows.

Step 1. Calculation of the order $p / q$ accumulation sequence

$$
X^{(p / q)}=\left\{x^{(p / q)}(1), x^{(p / q)}(2), \ldots, x^{(p / q)}(n)\right\} .
$$

Step 2. Put $x^{(p / q)}(k), k=1,2, \ldots, n$ into (19); then use the least squares estimate parameter of $\beta_{1}, \beta_{2}$, and $\beta_{3}$.

Step 3. Using

$$
\begin{array}{r}
\widehat{x}^{(p / q)}(k+1)=\beta_{1}^{k} \widehat{x}^{(p / q)}(1)+\beta_{2} \sum_{j=1}^{k} j \beta_{1}^{k-j}+\frac{1-\beta_{1}^{k}}{1-\beta_{1}} \beta_{3}, \\
k=1,2, \ldots, n-1,
\end{array}
$$

then we will predict $x^{(p / q)}(1), \widehat{x}^{(p / q)}(2), \ldots$

Step 4. Assume that $X^{(p / q)}=\left\{\hat{x}^{(p / q)}(1), \hat{x}^{(p / q)}(2), \ldots\right.$, $\left.\hat{x}^{(p / q)}(n)\right\}$, is the fractional order accumulation sequence and the equation

$$
\begin{aligned}
& \alpha^{(p / q)} X^{(0)}=\left\{\alpha^{(1)} \widehat{x}^{(1-p / q)}(1), \alpha^{(1)} \widehat{x}^{(1-p / q)}(2), \ldots,\right. \\
& \left.\alpha^{(1)} \widehat{x}^{(1-p / q)}(n+1), \ldots\right\}
\end{aligned}
$$

is established.

4.2. Optimizing the Order Number of the NDGM ${ }^{p / q}$ Model by Particle Swarm Optimization (PSO) Algorithm. The value of the mean absolute percentage error (MAPE) is often used to judge the merits of modeling. The order of the $\mathrm{NDGM}^{p / q}$ model is sought under the condition of the least mean absolute percent as follows:

$$
\min f(r)=\frac{1}{n-1} \sum_{k=2}^{n}\left|\frac{x^{(0)}(k)-\widehat{x}^{(0)}(k)}{x^{(0)}(k)}\right|, \quad r \in R^{+} .
$$

In this section, we will use the Particle Swarm Optimization (PSO) algorithm to optimize the order number of the $\mathrm{NDGM}^{p / q}$ model. The searing process of the optimization order of the $\mathrm{NDGM}^{p / q}$ model is as follows.

Step 1. Defining structure and initializing randomly the position (pBest) and speed for each particle, let $\mathrm{pBest}=1$.

Step 2. Set the first pBest $=1$ as the current position and gBest $=1$ as the optimal particle position in initial swarm.
Step 3. All the particles in the particle swarm were operated according to the following order:

(1) Update the locations and speeds of particles; let $C_{1}=$ $1, C_{2}=1$, and

$$
\begin{aligned}
P \cdot V= & V+C_{1} \times \text { rand } \times(\text { B Best }- \text { Present })+C_{2} \\
& \times \text { rand } \times(g \text { Best }- \text { Present }) \\
\text { Present }= & \text { Present }+V .
\end{aligned}
$$

(2) Judge whether the particle location Present meets the scope; if beyond the scope, a new location should be set for Present. If within the scope, then the calculation continued according to the following steps. When $r=p$ Best, the average relative error of fractional order operator model, the concrete steps were as follows:

(a) Computing $X^{(r)}, X^{(r)}$ is the rth order accumulating generating sequence of $X^{(0)}$.

(b) Compute the mean generated sequence with consecutive neighbours $Z^{(r)}$ of $X^{(r)}$.

(c) Compute the $r$-order inverse accumulating generation sequence $X^{(-r)}$ of $X^{(r)}$.

(d) Solve the parameters $\widehat{a}=\left[\beta_{1}, \beta_{2}, \beta_{3}\right]$.

(e) Deduce the time response expression of $\hat{x}^{(r)}(k)$.

(f) Compute the simulative values $\widehat{x}^{(r)}(k)$.

(g) Compute the restored simulative values $\widehat{x}^{(0)}(k)$ of $\widehat{x}^{(r)}(k)$.

(h) Compute the MAPE $f$ (pBest).

(i) Judge whether the value of $f(p$ Best) is less than $f$ (gBest); if $f(p B e s t)<f(g B e s t)$, the new position is set to pBest. If the $f(p B e s t)$ is not superior to gBest, the new position is set to gBest.

Step 4. Step 3 was carried out according to the order from the second particle to the last one.

Step 5. Judging whether the algorithm meets the convergence rule, if it meets then go to Step 6; else go to Step 3.

Step 6. Output Best "g," which is the optimal value of the order outputting the simulated or forecasted values of the NDGM $^{p / q}$ model, when $r=$ Best.

According to the modeling mechanism of the proposed $\mathrm{NDGM}^{p / q}$ model and the Particle Swarm Optimization (PSO) algorithm of the order number, the flowchart of the new model is summarized in Figure 2.

\subsection{Verification of $N D G M^{p / q}$ Model}

Case 2 (logistics demand forecasting in Jiangsu province [30]). We consider an example from paper [30] which provides the sample data. We will build four models that 


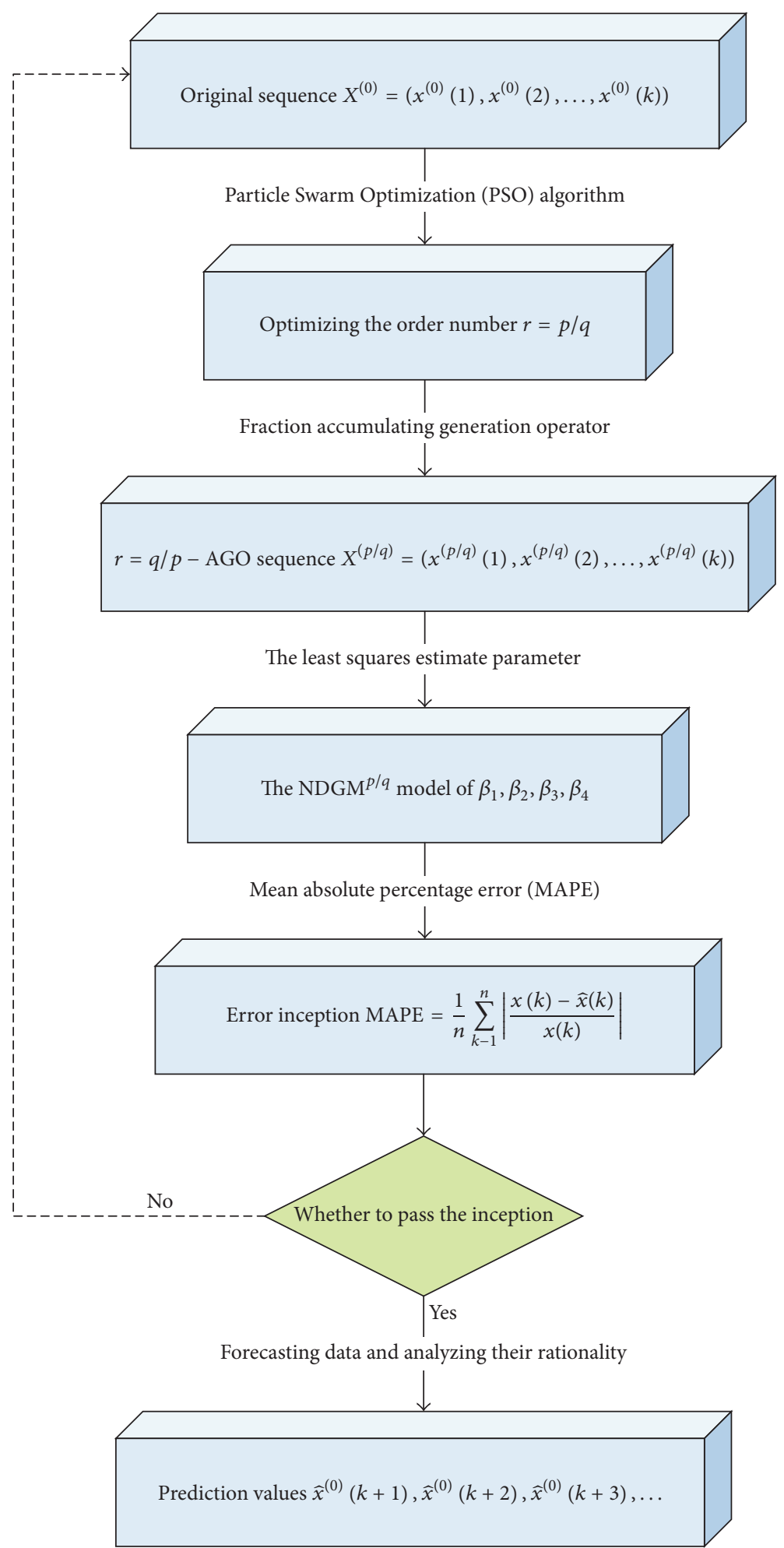

FIGURE 2: The flowchart of the NDGM ${ }^{p / q}$ model.

simulate Jiangsu province's logistics demand from 2002 to 2006 and to forecast it from 2007 to 2009 . We will compare the simulation and prediction mean absolute percentage error of the $\mathrm{NDGM}^{p / q}$ model with a classical NDGM model and DGM model.
According to MATLAB programs of the $\mathrm{NDGM}^{p / q}$ model and Particle Swarm Optimization (PSO) algorithm, the optimal order of the $\mathrm{NDGM}^{p / q}$ is $p / q=0.3298$. The simulated/forecasted values and mean absolute percentage error with the four different grey models are shown in Table 2. 


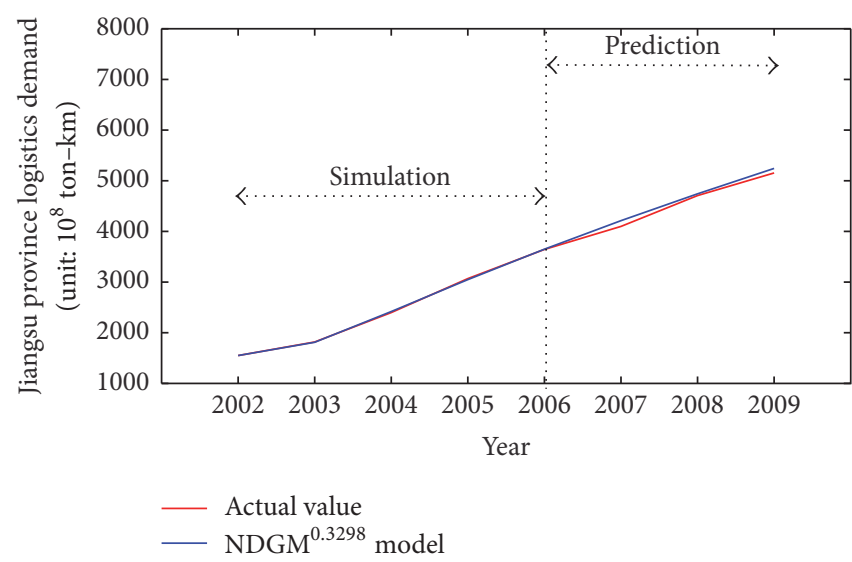

(a) The NDGM ${ }^{p / q}$ model

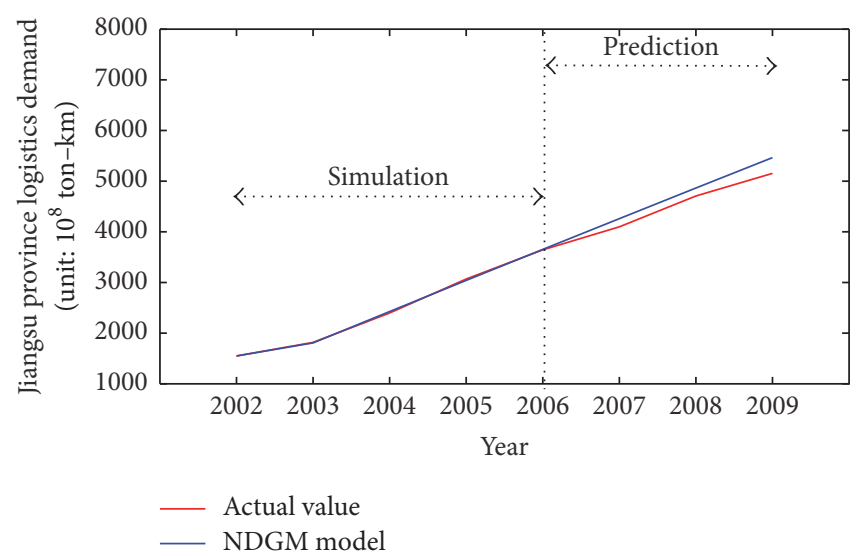

(b) The NDGM model

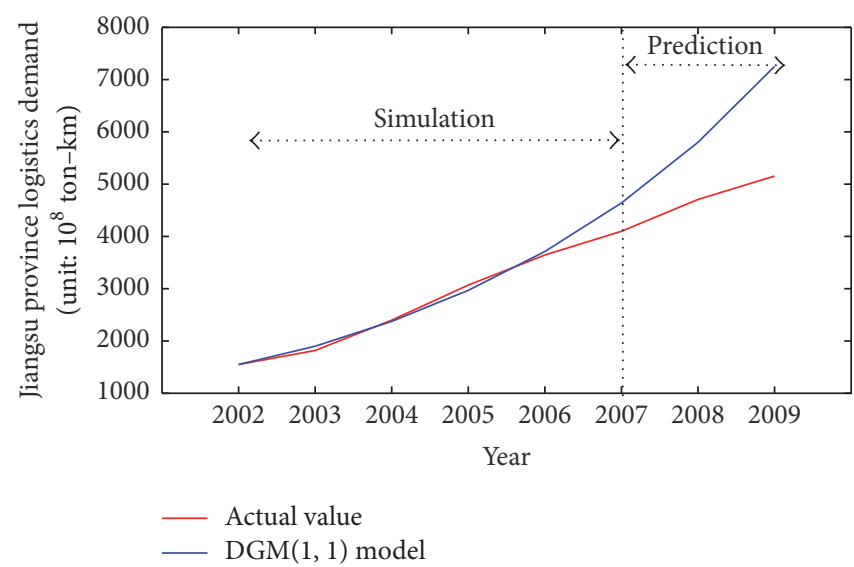

(c) The DGM model

FigURE 3

TABLE 2: The fitted values and MAPE of four grey models (unit: $10^{8}$ ton-km).

\begin{tabular}{lcccc}
\hline Year & Actual value & DGM & NDGM & NDGM $^{0.3298}$ \\
\hline 2002 & 1549.12 & 1549.12 & 1549.12 & 1549.12 \\
2003 & 1817.44 & 1899.10 & 1807.62 & 1811.13 \\
2004 & 2398.13 & 2374.15 & 2426.34 & 2418.20 \\
2005 & 3068.30 & 2968.18 & 3041.35 & 3048.45 \\
2006 & 3644.14 & 3710.85 & 3652.70 & 3648.96 \\
MAPE & & 2.65 & 0.71 & 0.49 \\
2007 & 4098.42 & 4639.35 & 4260.39 & 4212.31 \\
2008 & 4707.50 & 5800.16 & 4864.46 & 4741.86 \\
2009 & 5154.46 & 7251.42 & 5464.90 & 5242.83 \\
MAPE & & 25.70 & 4.44 & 1.74 \\
\hline
\end{tabular}

According to Table 2, in order to show the performances of four models clearly, we can draw Figures 3(a), 3(b), and $3(\mathrm{c})$.

As can be seen from Figures 3(a)-3(c), obviously, both of the simulation and prediction performance of the grey model with the optimal fractional order were superior to those of the corresponding grey model with an integer order. This showed that the fractional order accumulating generation could really improve the performance of a grey prediction model. It was shown that the improved effect of the fractional order on grey prediction model was also influenced by the raw model. Moreover, we can rank the effect of three models from the worst to the best in terms of their abilities to fit the data, namely, DGM model, NDGM model, and NDGM ${ }^{p / q}$ model.

\section{Conclusion}

When some systems were studied, the stability must be considered. The grey theory model mainly deals with the incomplete information cases and the uncertainty of the system models. From the perspective of model stability, $\mathrm{NDGM}^{p / q}$ model is more stable than traditional NDGM, and it was the result of a case to explain the stability. Because $\operatorname{GM}(1,1)$ model, DGM $(1,1)$ model, and NDGM model had a higher requirement for data and when the data did not meet the requirements, the errors of both simulation and prediction were larger, and the $\mathrm{NDGM}^{p / q}$ model has the important significance for improving the performance of grey model. Furthermore, we provided the Particle Swarm Optimization algorithm which was employed to optimize the 
order number of $\mathrm{NDGM}^{p / q}$ model. Using a case studied in Section 3, it has been shown that the MAPE of the NDGM ${ }^{p / q}$ model was always the lowest among the four grey models.

\section{Conflicts of Interest}

The authors declare that there are no conflicts of interest regarding the publication of this paper.

\section{Acknowledgments}

This work was supported by National Natural Science Foundation of China (Grants nos. 71540027, 11671001, 71671135, and 51479151), Chongqing Frontier and Applied Basic Research Project (Grants nos. cstc2015jcyjA00034 and cstc2015jcyjA00015), and the Science and Technology Research Program of Chongqing Municipal Educational Committee (Grant no. KJ1600425), Chongqing University of Posts and Telecommunications' Innovative Training Program (Grant no. 201610617088X).

\section{References}

[1] Z. a. Pawlak, "Rough sets," International Journal of Computer and Information Sciences, vol. 11, no. 5, pp. 341-356, 1982.

[2] Z. Pawlak, J. Grzymala-Busse, R. Slowinski, and W. Ziarko, "Rough sets," Communications of the ACM, vol. 38, no. 11, pp. 88-95, 1995.

[3] L. A. Zadeh, "Fuzzy sets," Information and Control, vol. 8, no. 3, pp. 338-353, 1965.

[4] J. L. Deng, "Introduction to grey system theory," The Journal of Grey System, vol. 1, no. 1, pp. 1-24, 1989.

[5] J. L. Deng, Estimate and Decision of Grey System, Wuhan: Huazhong University of Science and Technology Press, 2002.

[6] B. Zeng and C. Li, "Forecasting the natural gas demand in China using a self-adapting intelligent grey model," Energy, vol. 112, pp. 810-825, 2016.

[7] Z. Lihua, D. Suliang, J. Butterworth, X. Ma, B. Dong, and A. Liu, "Grey forecasting model for active vibration control systems," Journal of Sound and Vibration, vol. 322, no. 4-5, pp. 690-706, 2009.

[8] X. P. Xiao and S. H. Mao, GreY Forecating and Decision Methods, Science press, Beijing, China, 2013.

[9] Z.-X. Wang, "An optimized Nash nonlinear grey Bernoulli model for forecasting the main economic indices of high technology enterprises in China," Computers \& Industrial Engineering, vol. 64, no. 3, pp. 780-787, 2013.

[10] M. Wu, "Using multivariate regression to analyze the inuencing factors of real estate prices," Guide Bus, vol. 7, p. pp, 2012.

[11] Y. Wang, Q. Liu, J. Tang, W. Cao, and X. Li, "Optimization approach of background value and initial item for improving prediction precision of $\operatorname{GM}(1,1)$ model," Journal of Systems Engineering and Electronics, vol. 25, no. 1, Article ID 6754230, pp. 77-82, 2014.

[12] G. Tian, N. Li, and S. F. Liu, "Grey forecasting of Logistics demand based on parameter opti-mize model," East China Economy, vol. 2, pp. 155-157, 2011.

[13] L.-H. Chen and T.-Y. Guo, "Forecasting financial crises for an enterprise by using the Grey Markov forecasting model," Quality and Quantity, vol. 45, no. 4, pp. 911-922, 2011.
[14] Z. Zhao, J. Wang, J. Zhao, and Z. Su, "Using a grey model optimized by differential evolution algorithm to forecast the per capita annual net income of rural households in China," Omega, vol. 40, no. 5, pp. 525-532, 2012.

[15] Y. Wang, Y. Dang, Y. Li, and S. Liu, "An approach to increase prediction precision of $\mathrm{GM}(1,1)$ model based on optimization of the initial condition," Expert Systems with Applications, vol. 37, no. 8, pp. 5640-5644, 2010.

[16] Z. X. Wang, Y. G. Dang, and S. F. Liu, "An optimal GM(1,1) based on the discrete function with exponential law," Systems Enginnering-Theory Practice, vol. 28, no. 2, pp. 61-67, 2008.

[17] Z. X. Wang, Y. G. Dang, and S. F. Liu, "The optimization of background value in GM(1,1) model," The Journal of Grey System, vol. 10, no. 2, pp. 69-74, 2007.

[18] Y. Wei and D. H. Hu, "Deffciency of the smoothness condition and its remedy," Systems Engineering-Theory Practice, vol. 29, pp. 165-170, 2009.

[19] H.-L. Wong and J.-M. Shiu, "Comparisons of fuzzy time series and hybrid grey model for non-stationary data forecasting," Applied Mathematics \& Information Sciences, vol. 2, pp. 409416, 2012.

[20] N. M. Xie and S. F. Liu, "Discrete $\operatorname{GM}(1,1)$ and mechanism of grey forecasting model," Systems Engineering-Theory practice, vol. 25, no. 1, pp. 93-99, 2005.

[21] N. M. Xie and S. F. Liu, "Research on the non-homogenous discrete grey model and its parame-ter's properties," Journal of Systems Engineering and Electronics, vol. 30, no. 5, pp. 863-867, 2008.

[22] N.-M. Xie, S.-F. Liu, Y.-J. Yang, and C.-Q. Yuan, “On novel grey forecasting model based on non-homogeneous index sequence," Applied Mathematical Modelling, vol. 37, no. 7, pp. 5059-5068, 2013.

[23] N. Xie and S. Liu, "Interval grey number sequence prediction by using non-homogenous exponential discrete grey forecasting model," Journal of Systems Engineering and Electronics, vol. 26, no. 1, pp. 96-102, 2015.

[24] S. Mao, M. Gao, X. Xiao, and M. Zhu, "A novel fractional grey system model and its application," Applied Mathematical Modelling, vol. 40, no. 7-8, pp. 5063-5076, 2016.

[25] L. Wu, S. Liu, L. Yao, S. Yan, and D. Liu, "Grey system model with the fractional order accumulation," Communications in Nonlinear Science and Numerical Simulation, vol. 18, no. 7, pp. 1775-1785, 2013.

[26] L. F. Wu, S. F. Liu, Y. J. Yang et al., "Grey double exponential smoothing model and its application on pig price forecasting in China," Applied Soft Computing Journal, vol. 39, pp. 117-123, 2016.

[27] L. F. Wu, S. F. Liu, Y. J. Yang, L. Ma, and H. Liu, "Multi-variable weakening buffer operator and its application," Information Sciences, vol. 339, pp. 98-107, 2016.

[28] L.-F. Wu, S.-F. Liu, W. Cui, D.-L. Liu, and T.-X. Yao, "Nonhomogenous discrete grey model with fractional-order accumulation," Neural Computing and Applications, vol. 25, no. 5, pp. 1215-1221, 2014.

[29] S. F. Liu and Y. Liu, Grey Systems: Theory and Applications, Springer, London, Britain, 2006.

[30] L. F. Wu, S. F. Liu, L. G. Yao, and S. Yan, "The effect of sample size on the grey system model," Applied Mathematical Modelling, vol. 37, no. 9, pp. 6577-6583, 2013.

[31] J. G. Sun, Matrix Perturbation Analysis, Science Press, Beijing, China, 1987. 


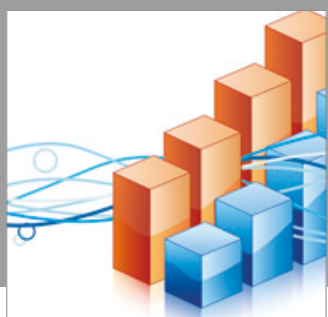

Advances in

Operations Research

vatersals

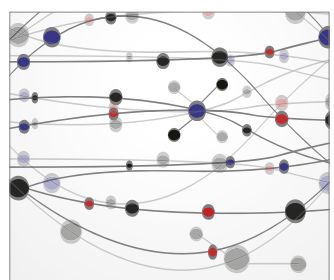

\section{The Scientific} World Journal
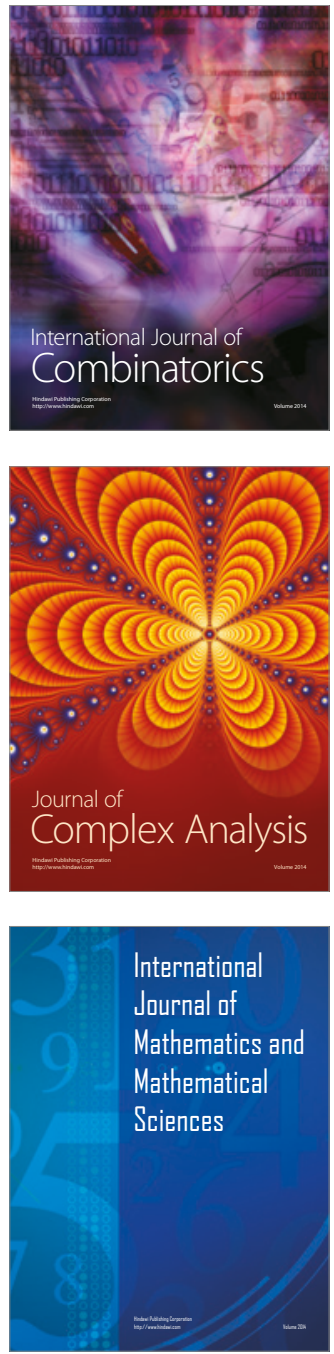
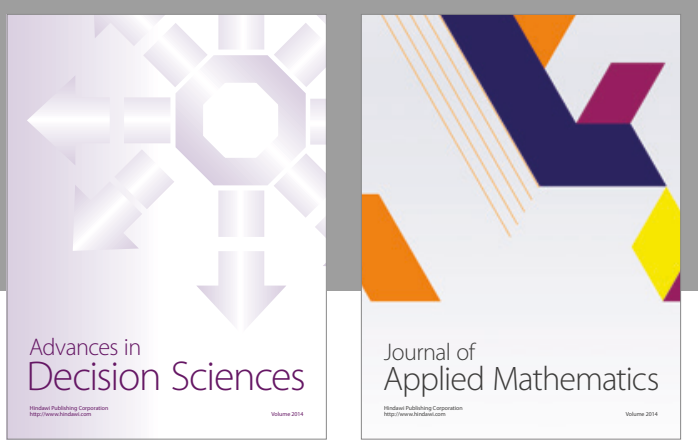

Algebra

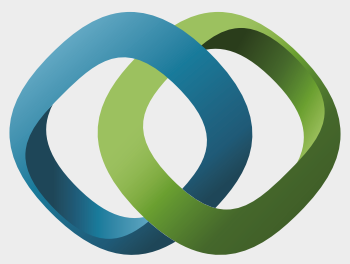

\section{Hindawi}

Submit your manuscripts at

https://www.hindawi.com
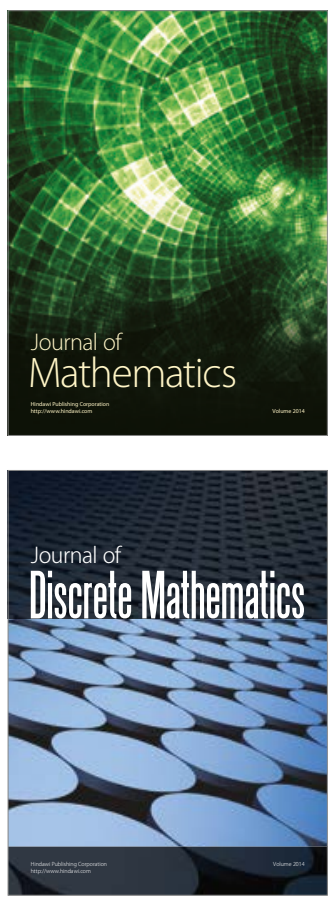

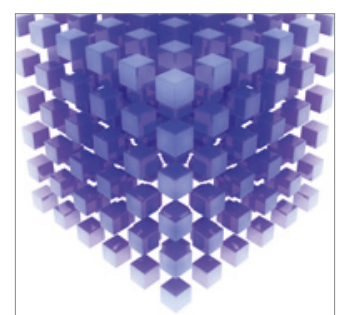

Mathematical Problems in Engineering
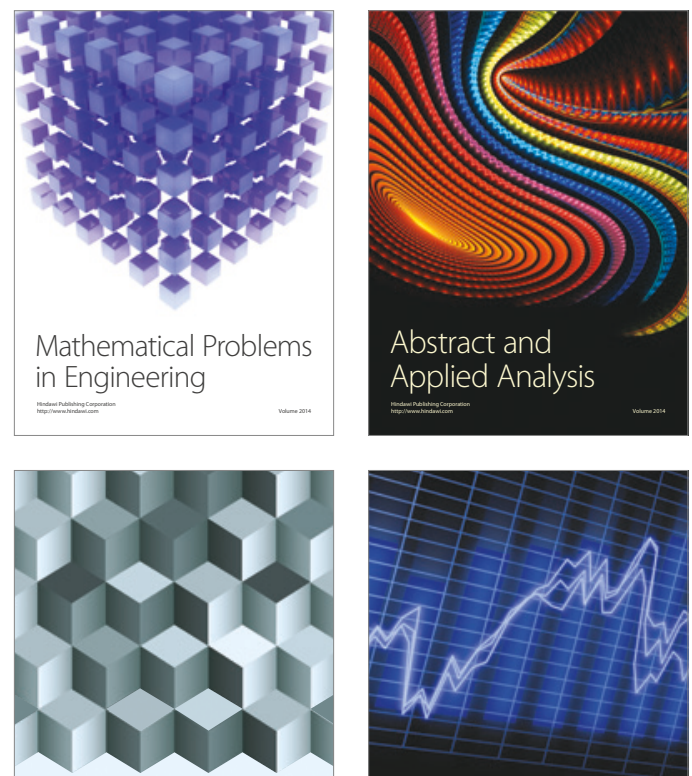

Journal of

Function Spaces

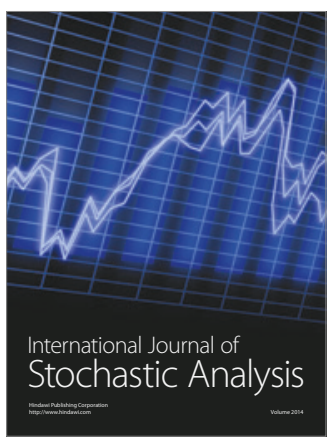

Probability and Statistics
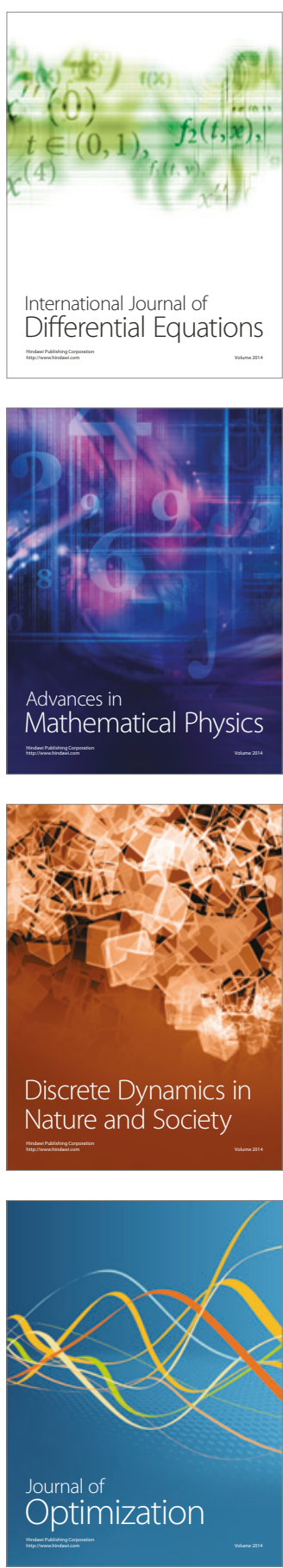